\title{
Radio relic and the diffuse emission trail discovered in low-mass galaxy cluster Abell 1697
}

\author{
Surajit Paul ${ }^{1}$, Sameer Salunkhe ${ }^{1}$, Satish Sonkamble ${ }^{2}$, Prateek Gupta ${ }^{1}$, \\ Tony Mroczkowski ${ }^{3}$, and Somak Raychaudhury ${ }^{4}$ \\ 2 National Centre for Radio Astrophysics, Tata Institute of Fundamental Research, Post Bag 3, S. P. Pune University Campus,
}

1 Department of Physics, Savitribai Phule Pune University, Pune 411007, India e-mail: surajit@physics.unipune.ac.in Ganeshkhind, Pune 411007, Maharashtra, India

3 European Southern Observatory (ESO), Karl-Schwarzschild-Str. 2, 85748 Garching, Germany

${ }^{4}$ Inter University Centre for Astronomy and Astrophysics, Pune 411007, India

Received 28 August 2019 / Accepted 31 October 2019

\begin{abstract}
We report the discovery of a putative radio relic, $830 \mathrm{kpc}$ in length, and found towards the outskirts of galaxy cluster Abell 1697 $(z=0.181)$, using the LOFAR Two Meter Sky Survey at $144 \mathrm{MHz}$. An X-ray-inferred mass of $M_{500}^{\mathrm{X} \text {-ray }}=2.9_{-0.7}^{+0.8} \times 10^{14} M_{\odot}$ places Abell 1697 among the least massive relic hosts. The relic is also detected at $325 \mathrm{MHz}$ in the Westerbork Northern Sky Survey and at $1.4 \mathrm{GHz}$ in the NRAO VLA Sky Survey, with an average spectral index of $\alpha(144,325,1400 \mathrm{MHz})=-0.98 \pm 0.01$, and magnetic field of $B_{\text {eq }} \sim 0.6 \mu \mathrm{G}$. This relic, located in the north-east periphery of the cluster, is $300 \mathrm{kpc}$ wide, exhibits a gradual spectral steepening across the width $\left(\alpha_{144 \mathrm{MHz}}^{1.4 \mathrm{GH}}(\mathrm{inj})=-0.70 \pm 0.11\right.$ to $\left.\alpha_{144 \mathrm{MHz}}^{1.4 \mathrm{GHz}}(\mathrm{edge})=-1.19 \pm 0.15\right)$, as well as indications of a co-spatial X-ray (ROSAT) shock and the radio relic emission. The radio power of the relic is $P_{1.4 \mathrm{GHz}}=8.5 \pm 1.1 \times 10^{23} \mathrm{~W} \mathrm{~Hz}^{-1}$, which is found to be in good agreement with the expected empirical correlation between the radio power and largest linear size of relics. The relic is trailed by extended $(790 \times 550 \mathrm{kpc})$ diffuse radio emission towards the cluster centre, which is likely an ultra-steep spectrum $\left(\alpha_{144 \mathrm{MHz}}^{1.4 \mathrm{GHz}}<-1.84\right)$ radio source. This structure is also found to be older by at least $190 \mathrm{Myr}$, has a very low surface brightness of $0.3 \mu \mathrm{Jy} \operatorname{arcsec}^{-2}$ and magnetic field $B_{\text {eq }} \sim 0.8 \mu \mathrm{G}$, similar to that of a radio phoenix. Finally, we discuss the possible mechanisms responsible for the relic and the trailing diffuse radio emission, invoking re-acceleration due to wake turbulence, as well as the revival of fossil electrons in the remnant radio lobes of active galactic nuclei by the cluster merger shocks.
\end{abstract}

Key words. radiation mechanisms: non-thermal - galaxies: clusters: individual: Abell 1697 - large-scale structure of Universe $\mathrm{X}$-rays: galaxies: clusters - radio continuum: general

\section{Introduction}

With the improved sensitivity of radio telescopes in recent years, diffuse and elongated radio sources, usually known as radio relics, are observed in a growing number of galaxy clusters (cf. review by van Weeren et al. 2019). The largest among these $(\gtrsim 1 \mathrm{Mpc})$ are the bow-shock relics, which occur at the peripheries of galaxy clusters. They have a low-surface brightness of $\sim 0.1-1 \mu \mathrm{Jy} \operatorname{arcsec}^{-2}$ at $1.4 \mathrm{GHz}$, are highly polarised (20-30\%), and are thought to originate from non-thermal synchrotron emission (e.g. van Weeren et al. 2019). These spectacular objects can be of two major types: symmetric doubles (e.g. Abell 3376, Bagchi et al. 2006), or single bow-like relics (e.g. Abell 2744, Paul et al. 2019) termed as "cluster relic shocks" (van Weeren et al. 2019) because of the strong evidence in favour of their association with cluster merger shocks.

Galaxy cluster mergers are the most energetic events in the Universe since the Big Bang itself, releasing a tremendous amount $\left(\sim 10^{64} \mathrm{ergs}\right)$ of energy (Sarazin 2002$)$ within a time span of $\gtrsim 1 \mathrm{Gyr}$. This creates an extreme pressure gradient in the intra-cluster medium (ICM), causing expansion at supersonic speeds (Paul 2012), and producing strong Mpc-scale shocks that travel across the cluster medium, reaching the cluster periphery. In the process, they dissipate energy by heating and generating turbulence in the ICM (Kang et al. 1996; Paul et al. 2011). These collision-less shocks are also known for efficiently accelerating charged particles via diffusive shock acceleration (DSA; Drury 1983) and magnetic field amplifiers (Iapichino \& Brüggen 2012), and eventually producers of radio emission through synchrotron process, creating radio relics (Kang et al. 2012). Alternatively, merger shocks can also reaccelerate fossil or ghost electrons from the past events of radio galaxies through compression, producing relic emissions called the phoenix (Enßlin \& Gopal-Krishna 2001; Kang et al. 2012).

Relativistic electrons lose energy very quickly through synchrotron emission and inverse Compton cooling, with an average life of about $10^{6}$ years, indicating a surviving structure of only about $100 \mathrm{kpc}$ (Kang et al. 2017). But, the radio structures in question are about a Mpc in size and remains visible for Gyr. Therefore, to keep a radio structure of an order larger in size and time-scale alive, the constant injection of relativistic electrons is absolutely necessary. Cluster merger shocks are the in-situ particle accelerators, reputedly injecting relativistic electrons continuously (Enßlin et al. 1998), keeping the bow-shock radio relics alive for longer ( $>$ Gyr). These shocks are analogous to the tsunami waves, becoming stronger (increasing in Mach number) as they reach the cluster periphery (Enßlin 2006; Paul et al. 2011), efficiently accelerating charged particles at the cluster 
outskirts through DSA mechanism, making radio emission better visible as cluster peripheral relics. Since particle acceleration happens only at a small width near to the shock front, the spectral index of peripheral radio emission steepens behind the surface of injection due to synchrotron losses, or ageing. Therefore, the spectral index distribution observed across these relics typically show a flat spectrum at the outer edge that gradually steepens towards the inner edge (Stroe et al. 2014). Radio phoenixes, by contrast, result from the re-energisation of the aged electron population present in the remnant lobes of an active galactic nuclei (AGN) by low Mach number shocks (de Gasperin et al. 2015), thus producing relic radio emission with extremely steep spectrum with curvature. They can be found both in the central region and the outskirts of the clusters (Hoeft et al. 2004).

Relics are detected more commonly in massive merging systems (de Gasperin et al. 2014). Low-mass clusters and groups are, however, more affected by mergers and various nongravitational processes (Lovisari et al. 2015) and contain high levels of cosmic-rays (Jubelgas et al. 2008), likely due to frequent AGN feedback events (Gilmour et al. 2007; Sivakoff et al. 2008). Since the sound speed in the ICM depends on ICM temperature, a merger of given velocity can produce a much higher Mach number shock in deeper-core ICMs of low-mass and low-temperature clusters than in higher mass clusters (Sarazin 2002). This would mean, if all relics are shock-generated, their fraction may be observed to increase in low-mass systems as the sensitivity of radio and X-ray surveys improve. But, so far, only a few relics have been reported to be found in lowmass clusters (de Gasperin et al. 2014, 2017; Kale et al. 2017; Dwarakanath et al. 2018).

We made the serendipitous discovery of a large diffuse radio relic emission in the low-mass $\left(M_{500}<5 \times 10^{14} M_{\odot}\right)$ cluster Abell 1697 while searching the field of cluster Abell 1682 in the LOFAR Two Meter Sky Survey (LoTSS). With further inspection, the relic-like emission in the cluster outskirts is also detected at $325 \mathrm{MHz}$ in the Westerbork Northern Sky Survey (WENSS), and at the high frequency of $1.4 \mathrm{GHz}$ in NRAO VLA Sky Survey (NVSS) maps. Here, in this work, we present a multi-wavelength study using the radio LoTSS, WENSS, NVSS, TGSS-ADR and VLA First survey, ROSAT survey in X-ray and Pan-STARRS1 optical survey data to report this discovery. We introduce the paper in Sect. 1. Detailed multiwavelength properties of the cluster are presented in Sect. 2. We discuss our findings and conclude the paper in Sects. 3 and 4, respectively.

We assumed $\Lambda$ CDM cosmology with parameters $H_{0}=70.2$, $\Omega_{\mathrm{M}}=0.3, \Omega_{\Lambda}=0.7$ for this study. The redshift of the cluster is $z=0.181$, yielding a luminosity distance $D_{\mathrm{L}}=869.2 \mathrm{Mpc}$ and angular scale of $3.026 \mathrm{kpc}$ per arcsecond.

\section{Cluster Abell 1697}

Abell 1697, also known as RXC J1313.1+4616, is a cluster in the northern sky at 13:13:04.56, +46:15:52.7 (J2000). This moderately distant cluster at a spectroscopic redshift of $z=0.1813 \pm$ 0.0006 (Clerc et al. 2016) hosts 84 galaxies within a radius of nine arcmin (Gal-Yam et al. 2008). Its colour-dependent stellar mass is reported to be $1.32 \pm 0.31 \times 10^{13} M_{\odot}$ (Sharon et al. 2007), while the total SZ mass is $M_{500}^{\mathrm{SZ}}=4.34_{-0.33}^{+0.32} \times 10^{14} M_{\odot}$ (Planck Catalogue: PSZ2 G111.75+70.37, Planck Collaboration XXIV 2016). The cluster has an $X$-ray brightness of $1.5 \pm 0.3 \times$ $10^{44} \mathrm{erg} \mathrm{s}^{-1}$ (0.1-2.4 keV, ROSAT survey, Clerc et al. 2016). While no X-ray estimated mass is reported yet, we provide estimates based on luminosity-mass scaling relations in Sect. 2.2.

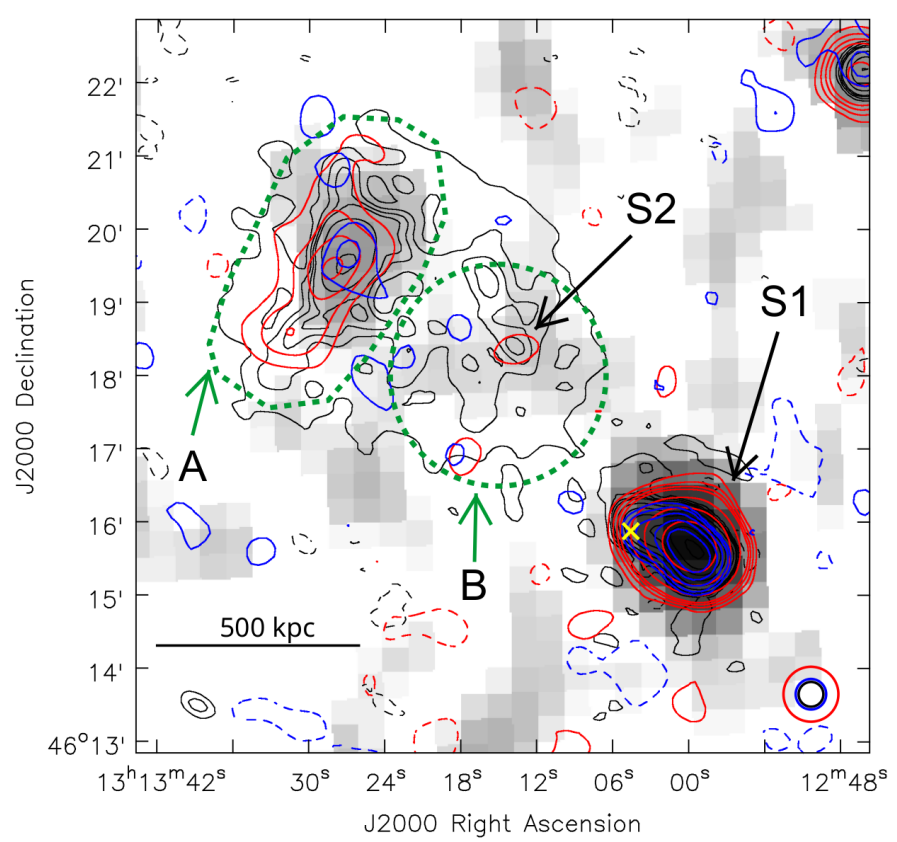

Fig. 1. Contours of low resolution $\left(20^{\prime \prime} \times 20^{\prime \prime}\right)$ LoTSS at $144 \mathrm{MHz}$ (black), NVSS at $1.4 \mathrm{GHz}$ (red) and TGSS at $150 \mathrm{MHz}$ (blue) on WENSS $(325 \mathrm{MHz})$ grey colour map. Contours are at $-3,5,9,13,17$, $34,68,136,272,544,1088 \times \sigma$ for LoTSS with $\sigma=150 \mu \mathrm{Jy}$, NVSS at $-3,3,5,7,9,18,36,72,144 \times \sigma$ with $\sigma=350 \mu \mathrm{Jy}$ and TGSS at $-3,3,5,10,20,40,80,160 \times \sigma$ with $\sigma=3 \mathrm{mJy}$. The relic and the trailing radio emission are highlighted by green dashed polygon ("A") and a circle ("B"), respectively. "S1" and "S2" are two point-like radio sources inside the cluster. The Abell cluster centre is marked as a yellow cross.

\subsection{Radio signatures}

In the field of Abell 1697, no diffuse radio relic or halo emission was previously reported. We report the newly-discovered extended diffuse radio emission using LoTSS, Data Release 1 (Shimwell et al. 2019). LoTSS provides a very deep image with root mean square noise (rms) $\sigma=150 \mu \mathrm{Jy}_{\text {beam }}^{-1}$ at $144 \mathrm{MHz}$. The black contour map in Fig. 1 shows the diffuse emission above $5 \sigma$. The region demarcated as " $\mathrm{A}$ " resembles peripheral relic structure. The proposed relic is also observed at $325 \mathrm{MHz}$ WENSS (grey) and at $1.4 \mathrm{GHz}$ NVSS (red contour), and only the brightest part is visible in TGSS-ADR 1 at $150 \mathrm{MHz}$ (blue contour). This relic is also attached to an extended trailing diffuse radio emission (as seen in LoTSS contour) towards the cluster centre, marked as "B".

In Fig. 2, we overlaid radio contours of LoTSS on PanSTARRS1 optical image, which shows that the extended radio emission on the north-east (NE) side of the cluster cannot be linked to any point sources, confirming its diffuse nature. The central radio source ("S1") that appears to be point-like in NVSS and WENSS, and extended in TGSS as well as in LoTSS maps, is resolved in VLA FIRST survey (see red contours in Fig. 2, inset). Apparently, it consists of a radio double and a radio galaxy towards the east of the double source. These radio point sources are found to be well-correlated with their optical counterparts. A diffuse optical emission can also be noticed just behind the relic front. A further investigation with GALEX near and far ultraviolet (UV) images and reported HII emission (Strobel et al. 1991) indicate a possible star-forming region. This region is far behind the peripheral radio relic structure, ruling out any physical connection between these two emissions. 
Table 1. Radio properties of diffuse radio structures (a) and radio point sources (b) found in the Abell 1697 galaxy cluster.

\begin{tabular}{|c|c|c|c|c|c|c|c|}
\hline \multicolumn{8}{|c|}{ (a) Diffuse radio structures } \\
\hline \multirow{2}{*}{$\begin{array}{l}\text { Survey } \\
\text { (Frequency }(\mathrm{MHz}) \text { ) }\end{array}$} & \multirow{2}{*}{$\begin{array}{c}\text { rms } \\
\left(\mu \mathrm{Jy} \mathrm{beam}^{-1}\right)\end{array}$} & \multirow{2}{*}{$\begin{array}{l}\text { beam } \\
\left({ }^{\prime \prime}\right) \\
\end{array}$} & \multicolumn{3}{|c|}{ Relic } & \multicolumn{2}{|c|}{ Trail (phoenix?) } \\
\hline & & & {$\left[\right.$ Size $\left({ }^{\prime \prime}\right)$} & Flux (mJy) & Power $\left.\left(\mathrm{W} \mathrm{Hz}^{-1}\right)\right]$ & [Size $\left({ }^{\prime \prime}\right)$ & Flux (mJy) ] \\
\hline LOFAR (144) & 150 & $20 \times 20, \mathrm{PA} 90^{\circ}$ & $275 \times 100$ & $97.1 \pm 9.8$ & $8.8 \pm 0.9 \times 10^{24}$ & $260 \times 180$ & $96.9 \pm 9.8$ \\
\hline TGSS-ADR1 (150) & 3000 & $25 \times 25$, PA $0^{\circ}$ & - & - & - & - & - \\
\hline WENSS (325) & 2000 & $54 \times 54, \mathrm{PA} 0^{\circ}$ & $142 \times 85$ & $34.9 \pm 3.5$ & $3.2 \pm 0.3 \times 10^{24}$ & - & - \\
\hline NVSS $(1.4 \mathrm{GHz})$ & 350 & $45 \times 45, \mathrm{PA} 0^{\circ}$ & $205 \times 75$ & $9.4 \pm 1.2$ & $8.5 \pm 1.1 \times 10^{23}$ & - & - \\
\hline \multicolumn{8}{|c|}{ (b) Radio point sources } \\
\hline \multirow[t]{3}{*}{ Survey } & \multicolumn{3}{|c|}{ Source "S1" } & \multicolumn{3}{|c|}{ Source "S2" } & \\
\hline & \multicolumn{2}{|c|}{ [Position } & Flux (mJy)] & \multicolumn{2}{|c|}{ [Position } & \multirow[t]{2}{*}{ Flux (mJy)] } & \\
\hline & (RA: & Dec:) & & (RA: & Dec:) & & \\
\hline LOFAR & $13: 12: 59.6$ & 46.15 .40 .3 & $449.0 \pm 44.9$ & $13: 13: 13.7$ & 46.18 .25 .0 & $3.6 \pm 0.4$ & \\
\hline TGSS-ADR1 & & & $413.2 \pm 42.0$ & & & - & \\
\hline WENSS & & & $307.8 \pm 31.2$ & & & - & \\
\hline NVSS & & & $51.1 \pm 5.2$ & & & $0.4 \pm 0.2$ & \\
\hline
\end{tabular}

Notes. (a) Names of surveys and their respective rms and beam size are given in first three columns. Size, flux density, and power of the proposed relic in the next three columns. Final two columns show the size and flux density of the trailing diffuse emission. (b) Position, flux and radio powers of each of the point sources (i.e. S1 and S2) are given in columns 2 and 7; respectively.

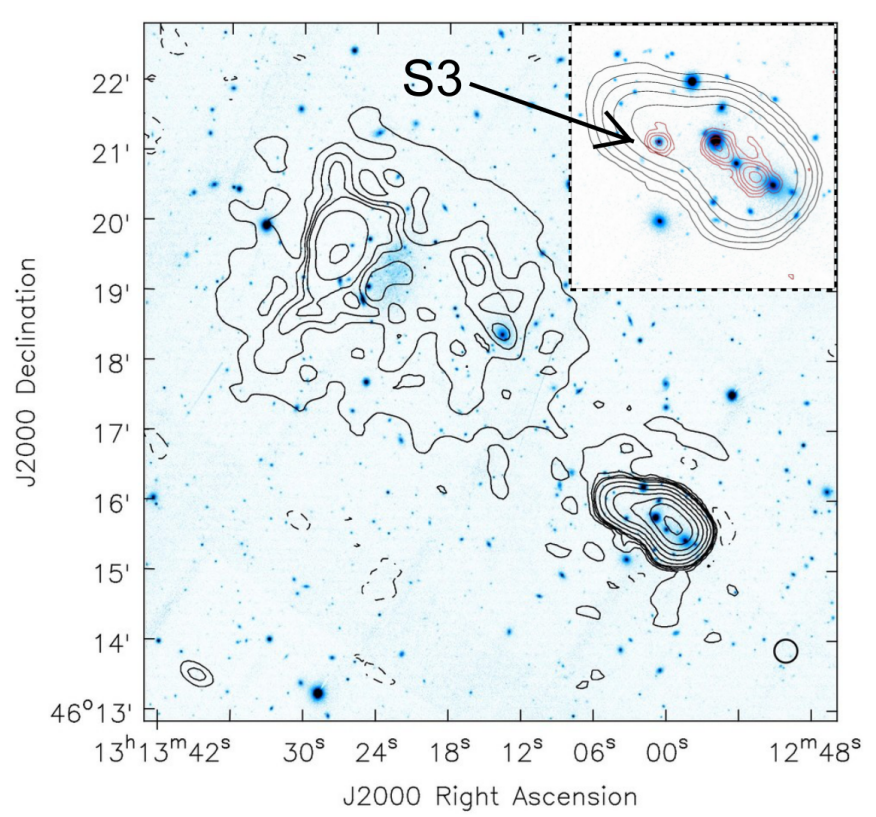

Fig. 2. Pan-STARRS1 “i” band optical image overplotted with LoTSS low-resolution contours (same as Fig. 1). Inset: high-resolution VLA First and LOFAR contours for the central radio source are overplotted on Pan-STARRS1 image. VLA First contours are at 3,6,12, 24, $48 \times$ $150 \mu \mathrm{Jy}$ and LoTSS contours are at $10,20,40,80,160 \times \sigma$. "S3" $(13: 13: 03.0,46.15 .48 .6)$ is the third radio point source in Abell 1697.

\subsubsection{Flux densities}

We computed the flux densities $(S)$ of observed radio sources within $3 \sigma$ contours ( $\sigma$ is noise rms of the images given in Table $1,5 \sigma$ for LoTSS) and flux density errors $\left(\sigma_{S}\right)$ using the usual relation,

$\sigma_{S}=\sqrt{(0.1 S)^{2}+N(\sigma)^{2}}$,

where $N$ is the number of beams covered by the total diffuse emission, and $0.1 S$ (i.e., $10 \%$ of $S$ ) was assumed as the possible error, due to calibration uncertainties.
The peripheral relic towards the NE of the cluster is a bright source at $144 \mathrm{MHz}$ with a flux density of $97.1 \pm 9.8 \mathrm{mJy}$ and size of $830 \times 300 \mathrm{kpc}$. The relic visible in the NVSS map is of $620 \times$ $225 \mathrm{kpc}$. It is a low-brightness object at $1.4 \mathrm{GHz}$ with a flux density of $9.4 \pm 1.2 \mathrm{mJy}$ and radio power of $8.5 \pm 1.1 \times 10^{23} \mathrm{~W} \mathrm{~Hz}^{-1}$. The large trailing emission behind the shock observed in LoTSS map ("B" in Fig. 1) is about $790 \mathrm{kpc}$ long and $550 \mathrm{kpc}$ in width, with a flux density of $96.9 \pm 9.8 \mathrm{mJy}$ (at $144 \mathrm{MHz}$ ). Except for a bright point source ("S2"), the trailing emission is not well-detected in the NVSS or WENSS maps, and nothing is observed in the TGSS map. The point-like source ("S1" in Fig. 1) observed in all the surveys is a very bright source at low frequency $(144 \mathrm{MHz})$ with a flux density of $449.0 \pm 44.9 \mathrm{mJy}$. Furthermore, detailed parameters measured at different observed frequencies are given in Table 1(a) and (b).

\subsubsection{Spectral properties}

The images were first re-gridded with IMREGRID task of CASA, and thereafter beams are convolved to the beam of $1.4 \mathrm{GHz}$ NVSS i.e. $45^{\prime \prime} \times 45^{\prime \prime}$ with the IMSMOOTH task. The UV coverage cannot be homogenised, as these are survey images from different telescopes. The images were then masked below $3 \sigma$ using the NVSS image. Finally, we computed the spectral index using CASA IMMATH task with the relation,

$\alpha=\frac{\log \left(S_{v_{2}} / S_{v_{1}}\right)}{\log \left(v_{2} / v_{1}\right)}$

where $S_{v_{\#}}$ and $v_{\#}$ (with $v_{1}>v_{2}$ ) are the flux densities and the respective observed frequencies. The spectral index error map is made using the relation given below (Kim \& Trippe 2014):

$\alpha_{\mathrm{err}}\left(\alpha_{v_{2}, v_{1}}\right)=\frac{1}{\log \left(v_{2} / v_{1}\right)} \times\left[\frac{\sigma_{v_{1}}^{2}}{I_{v_{1}}^{2}}+\frac{\sigma_{v_{2}}^{2}}{I_{v_{2}}^{2}}\right]^{\frac{1}{2}}$,

with $I$ as the total intensity at respective frequencies at each of the pixels.

The left panel of Fig. 3 shows a spectral index map of the studied radio features combining the images at $144 \mathrm{MHz}$ (LoTSS) 

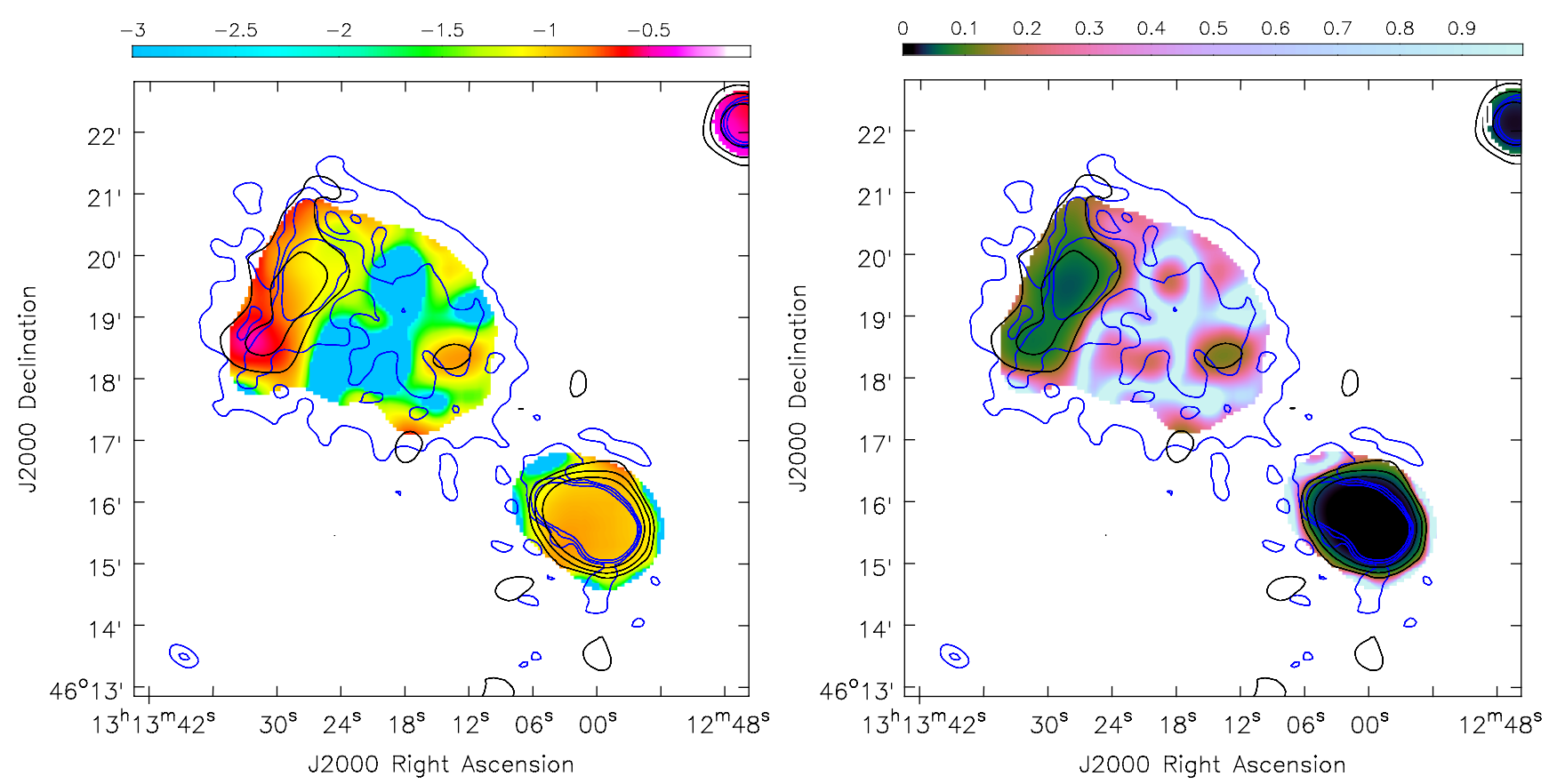

Fig. 3. Left panel: spectral index map using LOFAR $144 \mathrm{MHz}$ and NVSS $1.4 \mathrm{GHz}$. Right panel: spectral index error map for the same.

and 1.4 GHz (NVSS). The corresponding error map is plotted in the right panel of Fig. 3. The average spectral index of the proposed relic part (i.e. "A", with $3 \sigma$ detection of NVSS) is about $\alpha=-0.96 \pm 0.17$. The spectral index at the shock front (i.e., at injection) is $\alpha_{\mathrm{inj}}=-0.70 \pm 0.14$, which steepens across the relic gradually, and falls to $\alpha_{\text {edge }}=-1.19 \pm 0.23$. The average combined spectral index of the relic is found to be $\alpha(144,325,1400 \mathrm{MHz})=$ $-0.98 \pm 0.01$. Because of non-detection of the structure " $\mathrm{B}$ " in both NVSS and WENSS, we computed the upper limits using the local root-mean-square (rms) of $0.35 \mathrm{mJy} \mathrm{beam}^{-1}$ (NVSS) and $2 \mathrm{mJy} \mathrm{beam}^{-1}$ (WENSS), respectively, and we excluded the point source, marked as "S2" in Fig. 1. Upper limits for the spectral index computed are therefore $\alpha_{144 \mathrm{MHz}}^{1.4 \mathrm{GHz}}$ (trail) $<-1.84$ and $\alpha_{144 \mathrm{MHz}}^{330 \mathrm{MHz}}$ (trail) $<-2.02$. The average spectral index for the source " $\mathrm{S} 1$ " is computed as $\alpha_{144 \mathrm{MHz}}^{1.4 \mathrm{GHz}}=-0.96 \pm 0.02$. Using the relation of Mach number and the spectral index,

$\mathcal{M}^{2}=\frac{2 \alpha-3}{2 \alpha+1}$

(considering DSA mechanism; see Blandford \& Eichler 1987; Colafrancesco et al. 2017), we computed the average Mach number of the shock as $\mathcal{M}=2.31_{-0.27}^{+0.50}$ (using the average spectral index of $\alpha_{144 \mathrm{MHz}}^{1.4 \mathrm{GHz}}=-0.96 \pm 0.17$ ) for the proposed relic.

\section{2. $X$-ray properties}

Figure 4 shows the photon count map of the ROSAT survey at $0.1-2.4 \mathrm{keV}$. The grey colour represents the photon counts, and dark green contours indicate the mild Gaussian smoothed map. The NVSS Radio flux is overplotted as red contours. The ROSAT X-ray survey data is of just $0.6 \mathrm{ks}$ : inadequate to create any X-ray brightness or temperature maps. Nonetheless, the dark green contours of the mildly-smoothed photon counts show an overall asymmetric morphology, clumpy and extended towards the north-west. We also find that the photon count does not vary smoothly or decline monotonically.

The observed peak and extreme end of the radio relic-like diffuse emission (see red NVSS contours towards NE) are about

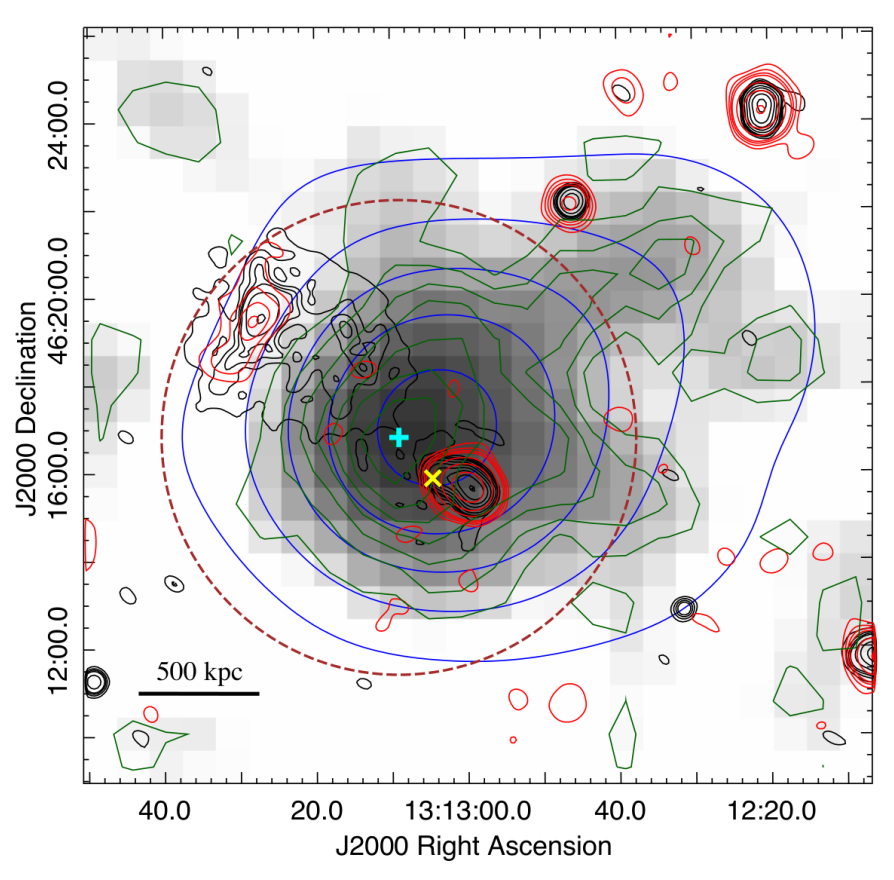

Fig. 4. ROSAT X-ray photon count map (0.1-2.4 keV) presented in grey, mildly and highly Gaussian smoothed contours are in dark green and blue, respectively. NVSS and LoTSS radio contours are shown in red and black, respectively. Red dashed circle represents $r_{500}$ radius, where the X-ray peak (centre) and the Abell cluster centre were marked as cyan "+" and yellow " $\times$ ", respectively.

$270^{\prime \prime}$ and $340^{\prime \prime}$ (i.e. about $815 \mathrm{kpc}$ and $1.02 \mathrm{Mpc}$, respectively) away from the X-ray peak or the X-ray centre (peak of mild Gaussian smoothed map at 13:13:08.6, +46:16:48.9, marked as cyan plus in Fig. 4). Furthermore, we plotted the radial profile of the photon count for a sector that encloses the radio relic in Fig. 5b. A dip in the profile, possibly indicating a shock front, appears at around $280 \pm 23^{\prime \prime}$ from the X-ray centre 

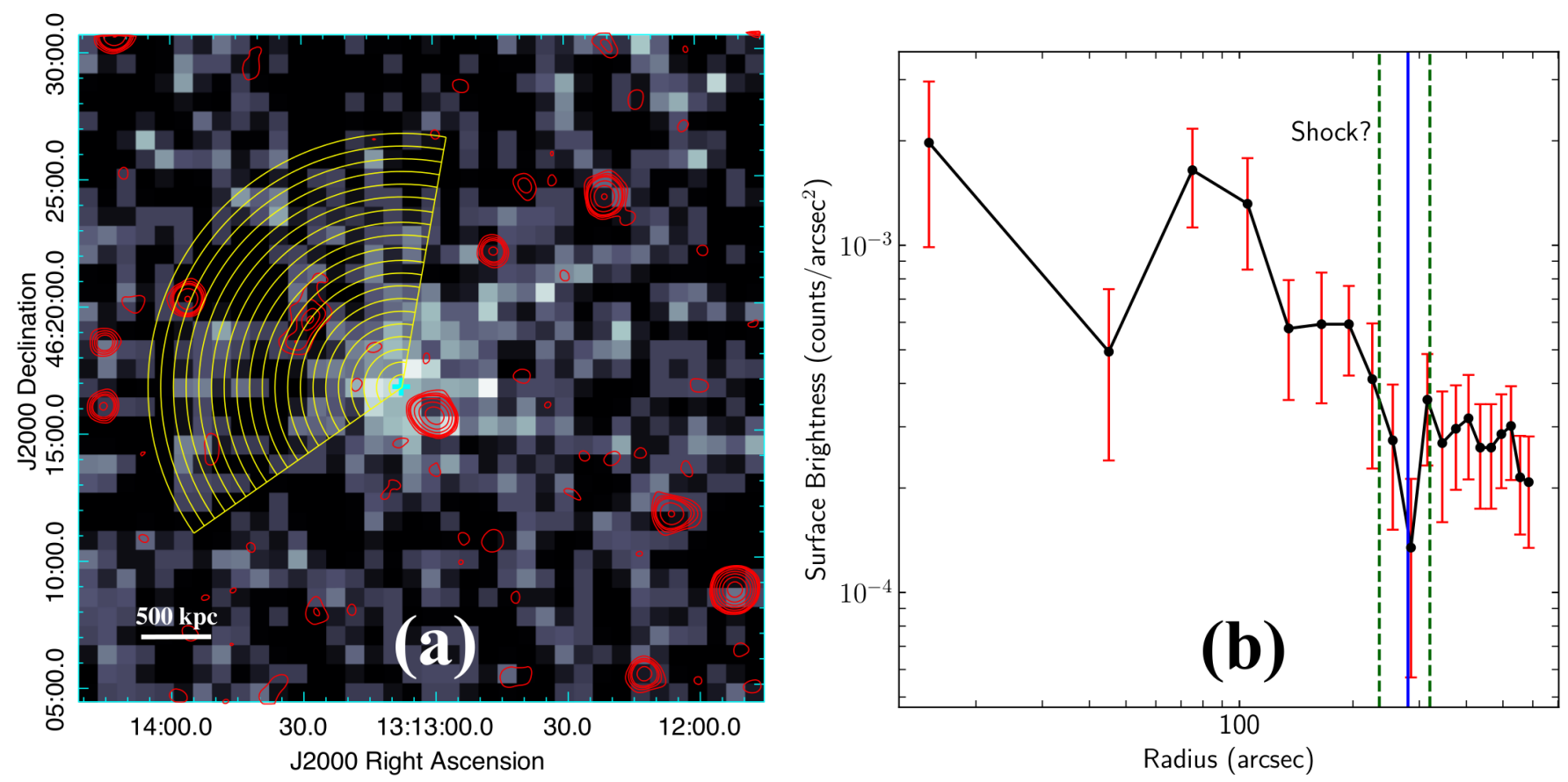

Fig. 5. Panel $a$ : sector for which radial profile of X-ray photon count (0.1-2.4 keV ROSAT survey data) is displayed in panel b. Panel $b$ : photon count radial profile for the sector containing the radio relic shown in panel a and the cluster radio shock position (blue vertical line). Distance of the inner and the outer edge of the radio relic (from the X-ray peak) are indicated with dark green vertical dashed lines.

(i.e. $850 \pm 70 \mathrm{kpc}$ from the Gaussian peak fit). This feature overlaps with the cluster radio shock (relic) and the shock front as shown in Fig. 5b with green vertical dashed-lines (at 235" and $320^{\prime \prime}$ i.e. $\sim 710-970 \mathrm{kpc}$ ). We caution the reader that ROSAT data have a large point spread function and low significance, making shock identification exceedingly difficult at moderate redshifts such as this one.

The reported X-ray luminosity for a photon energy range of $0.1-2.4 \mathrm{keV}$ (ROSAT) is $L_{500}=1.5 \pm 0.3 \times 10^{44} \mathrm{erg} \mathrm{s}^{-1}$ (SPIDERS clusters, Clerc et al. 2016). Adopting the method described by Mantz et al. (2018), Eq. (6), we estimated a mass of $2.9_{-0.7}^{+0.8} \times$ $10^{14} M_{\odot}$. The reported SZ mass of $M_{500}^{\mathrm{SZ}}=4.34_{-0.33}^{+032} \times 10^{14} M_{\odot}$ is in rough agreement with the mass computed from X-ray data. Using the virial radius relation derived from the self-similarity (Kaiser 1986), expressed as

$r_{\mathrm{vir}}=\left[3 M_{200} /\left(4 \pi \nabla_{\mathrm{c}}(z) \rho_{\mathrm{cr}}(z)\right)\right]^{1 / 3}$

(where $M_{200}$ is virial mass, $\nabla_{\mathrm{c}}(z)$ and $\rho_{\mathrm{cr}}(z)$ are the over density ratio and critical density at redshift $z$, respectively), we estimated the virial radius corresponding to the X-ray mass of $2.9 \times 10^{14} M_{\odot}$ as $r_{200}=1.3 \mathrm{Mpc}$, (i.e. $r_{500} \sim 1 \mathrm{Mpc}$; see Fig. 4). Furthermore, our estimate for the average temperature of the gas, $T_{\mathrm{g}}$, is $3.5 \mathrm{keV}$ (i.e. $4.0 \times 10^{7} \mathrm{~K}$ ) as calculated from the relation $T_{\mathrm{X}}=2.34 L_{44}^{1 / 2} h_{50}$ (Böhringer et al. 2000) using $L_{\mathrm{X}}=1.5 \times 10^{44} \mathrm{erg} \mathrm{s}^{-1}$ for uncorrected X-ray luminosity.

\section{Discussion}

The elongated, peripheral diffuse radio emission found in the field of cluster Abell 1697 has a largest linear size of $830 \mathrm{kpc}$ and width of $300 \mathrm{kpc}$ in the LoTSS map. The extreme end of the proposed relic emission is found $\sim 1 \mathrm{Mpc}$ away (i.e. close to $r_{500}$ ) from the X-ray peak (see Fig. 4). The emission is purely diffuse in nature as is evident from the radio and optical images

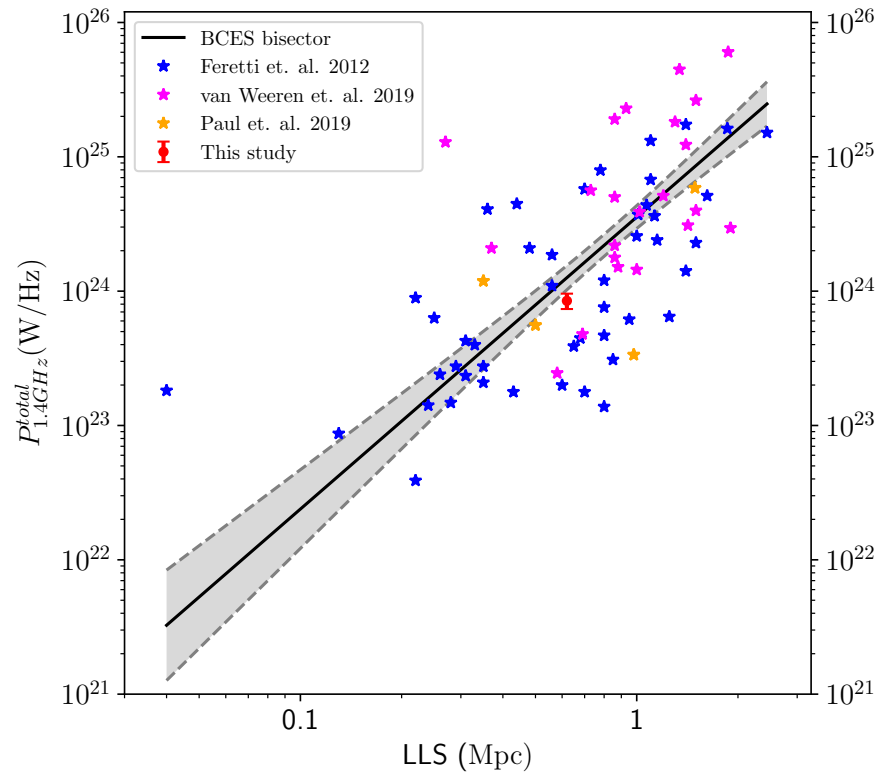

Fig. 6. BCES-Bisector fit for radio power $(1.4 \mathrm{GHz})$ vs. LSS correlation. Data collected from Feretti et al. (2012), Paul et al. (2019), van Weeren et al. (2019). Relic found in Abell 1697 is plotted as red point with error bars.

(see Sect. 2.1). The average spectral index estimated for this relic is relatively flat $\left(\alpha_{144 \mathrm{MHz}}^{1.4 \mathrm{GHz}}=-0.96 \pm 0.17\right)$, but well within the variations found in relics so far (van Weeren et al. 2019). The average equipartition magnetic field is estimated (see the method in Jamrozy et al. 2004) as $0.6 \mu \mathrm{G}$. The spectrum of the relic is flatter at the front, i.e. at injection $\left(\alpha_{\text {inj }}=-0.70 \pm .14\right)$, and gradually steepens to $\left(\alpha_{\text {edge }}=-1.19 \pm 0.23\right)$ at the inner edge, indicating a Compton synchrotron emission process with continuous injection (Bonafede et al. 2012), usually a characteristic 

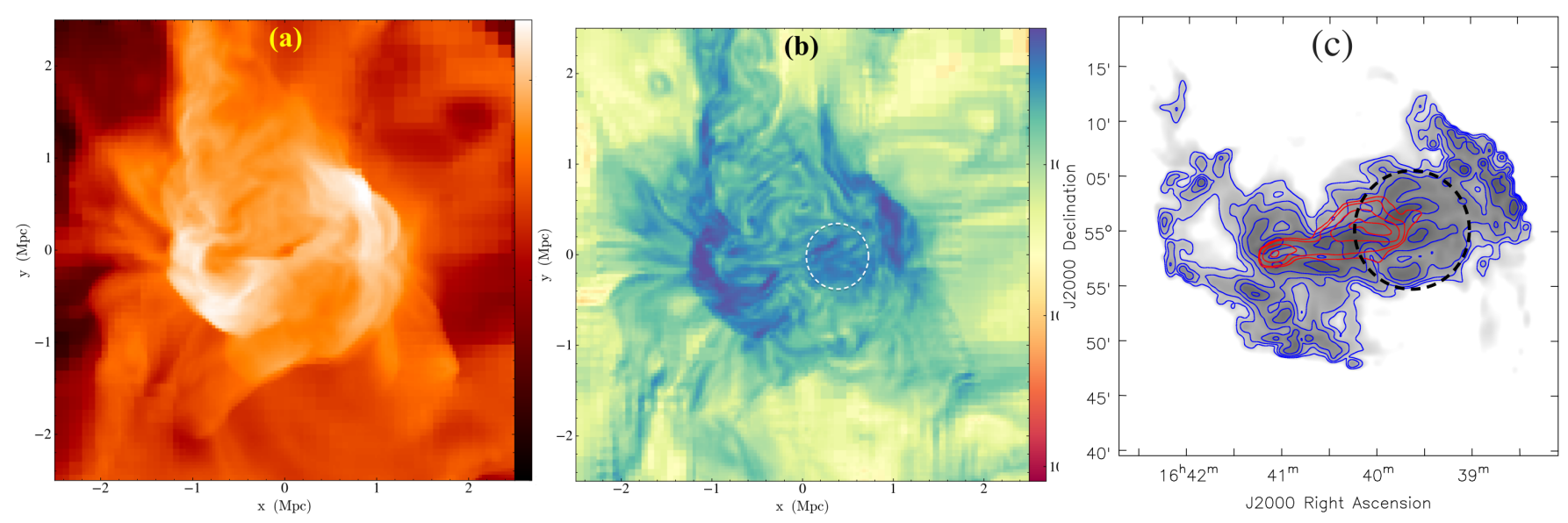

Fig. 7. Panels $a$ and $b$ : respectively post-merger temperature shock and vorticity magnitude $(\bar{\omega}=\nabla \times \bar{v}$ (representing turbulence) of a simulated cluster. Panel $c$ : modelled mock radio map considering radio emission from both DSA and TRA electrons (see Appendix A.1) plotted as grey (zoomed on the cluster). The computed X-ray luminosity is overlaid in red contours. Co-ordinates shown are arbitrary.

of peripheral relics. Furthermore, we found that the proposed relic perfectly follows the correlation of relic radio power (at $1.4 \mathrm{GHz}$ ) and its size (LLS) with a correlation slope of $2.17 \pm 0.25$ (see the plot in Fig. 6), first discussed in Feretti et al. (2012) and recently updated in Paul et al. (2019).

From the ROSAT survey X-ray map (see Fig. 4), we detect features indicative of ongoing dynamical activities in the cluster. The contours from the X-ray photon count map coincide well with the radio relic morphology (see Fig. 4 and Sect. 2.2). Also, strikingly, there is a dip in X-ray photon count observed in the profile map at a distance of $850 \pm 70 \mathrm{kpc}$ from the Xray peak. This potential shock is in the sector that encloses the radio relic (see Fig. 5b), and reasonably matches with the distance between the X-ray peak and the brightest radio emission (i.e. $815 \mathrm{kpc}$ ). The relic front (i.e. $970 \mathrm{kpc}$ ), as well as its extension $(\sim 710-970 \mathrm{kpc})$, indicate a connection between the peripheral radio emission and the X-ray shock. An inadequate photon count restricted us from making a temperature profile to confirm this connection.

\subsection{Turbulent re-acceleration by wake turbulence?}

We performed a cosmological simulation for a merging cluster using ENZo, an $\mathrm{N}$-body plus hydrodynamic, adaptive mesh refinement (AMR) code (Bryan et al. 2014). A realisation of a sky patch with a linear scale of $128 \mathrm{Mpc}(1+z)^{-1} h^{-1}$ [i.e. having a co-moving volume of $\left.\left(128 \mathrm{Mpc}^{-1}\right)^{3}\right]$ is produced in a flat $\Lambda \mathrm{CDM}$ background cosmology. The radio emission is computed from the simulated merging cluster, implementing a synchrotron emission mechanism invoking both the DSA and turbulent reconnection acceleration (TRA) as the electron injection models to understand the large radio emission found in Abell 1697. For more details on these simulation studies, as well as the time series of cluster merger events and its thermal and non-thermal energy evolution, see Appendix A.

In Fig. 7a, we see a post-merger thermal shock producing the wake turbulence behind it (see Fig. 7b). The simulated mock radio as well as X-ray map (using cLoudy code, Ferland et al. (2017)), shown in Fig. 7c, indeed show a possibility of finding a peripheral relic due to DSA along with a trailing radio emission extending till the cluster centre due to TRA, thus tentatively explaining the observed radio structures reported in this study.

\subsection{A radio lobe angle?}

Giant radio galaxies usually have two symmetric radio lobes. Also, the brightest emission observed inside the proposed relic does not resemble the hot-spots of a radio galaxy in morphology, magnetic field strength (much lower at $\sim 1.4 \mu \mathrm{G}$ ), as well the unusually steep spectrum $(\alpha<-1)$. The trailing diffuse emission shows an extremely steep average spectrum (upper limit) of $\alpha_{\text {trail }} \leq-1.84$ with an average magnetic field and age of the electrons computed as $0.8 \mu \mathrm{G}$ and $190 \mathrm{Myr}$ respectively (using the method of Jamrozy et al. 2004), hardly fits the characteristics of an active giant radio galaxy. Further, the brightest source (i.e. "S1" in Fig. 1) does not seem to be just a point source as indicated from its steep spectrum $(\alpha<-1)$. Indeed, the VLA FIRST map resolves it to two prominent radio sources (Fig. 2, inset). One is a bright radio double source with a central optical counterpart with a VLA FIRST $(1.4 \mathrm{GHz})$ flux density of $40.4 \pm$ $4.1 \mathrm{mJy}$. The two other optical sources observed near to each of the radio lobes do not match the radio peak positions. One among them in the NE direction is not even marked as a galaxy in NED. Therefore, the central radio source is a confirmed double source, surely not the core of a giant radio galaxy. The second radio source $\mathrm{S} 3$, is a very weak one with a VLA FIRST $1.4 \mathrm{GHz}$ radio power of $P_{1.4 \mathrm{GHz}}=5.24 \pm 1.26 \times 10^{23} \mathrm{~W} \mathrm{~Hz}^{-1}$, unlikely to host a giant radio source of size more than an Mpc (Lara et al. 2004).

\subsection{Is the trailing diffuse emission a radio phoenix?}

The other feasible situation is that the structure is a revived radio lobe. The spectral index upper limit of the trailing diffuse radio emission $\left(\alpha_{144 \mathrm{MHz}}^{1.4 \mathrm{GHz}}\right.$ (trail) $<-1.84$ and $\alpha_{144 \mathrm{MHz}}^{330 \mathrm{MH}}($ trail $\left.)<-2.02\right)$ indicates it likely falls in the category of ultra-steep spectrum radio sources. A cluster merger shock may have passed through the ghost electron clouds from the past AGN activity, and revived the fossil electrons by adiabatic shock compression, as in the scenario proposed by Enßlin \& Gopal-Krishna (2001), known as the radio phoenixes. The proposed phoenix has a large size of about $800 \times 550 \mathrm{kpc}$ with extremely faint radio surface brightness of $0.3 \mu \mathrm{Jy} \operatorname{arcsec}^{-2}$ at $144 \mathrm{MHz}$ LoTSS map. The estimated magnetic field strength is low, with an average of $\sim 0.8 \mu \mathrm{G}$, and the average electron age is $190 \mathrm{Myr}$. The distance of the phoenix centre to the shock front is about $500 \mathrm{kpc}$. With a shock speed of $\sim 2020 \mathrm{~km} \mathrm{~s}^{-1}$, as computed from the average shock Mach 
number of $\mathcal{M} \sim 2.3$ and the temperature of $\sim 3.5 \mathrm{keV}$ (from the relation $V_{\mathrm{sh}}=\mathcal{M}\left[1480\left(T_{\mathrm{g}} / 10^{8} \mathrm{~K}\right)^{1 / 2}\right] \mathrm{km} \mathrm{s}^{-1}$ (Sarazin 1988) where $T_{\mathrm{g}}$ is the temperature of the downstream ICM gas), the shock passage time is about $250 \mathrm{Myr}$, thus indicating a strong case of revival of fossil electrons to generate the trailing diffuse radio emission found in cluster Abell 1697 at $144 \mathrm{MHz}$ LoTSS maps.

\section{Conclusions}

We report the discovery of a relic-like diffuse radio emission from the outskirts of the cluster Abell 1697, as well as an extremely steep spectrum diffuse radio emission trailing behind the said relic. The LoTSS, NVSS, and WENSS radio maps, along with the X-ray map from the ROSAT All-Sky Survey, provide reasonable evidence that the structure is a peripheral relic. The trailing diffuse radio emission has very low surface brightness and an extremely steep spectrum, indicative of a radio phoenix.

We discuss various possibilities to understand the mechanism responsible for the observed radio structures invoking turbulent re-acceleration, as well as the process of revival of fossil electron clouds from an old AGN activity. However, no firm conclusions on its origin can be drawn, due to the absence of deep observations, mainly at high radio frequencies and in X-rays.

Acknowledgements. This research was funded by DST INSPIRE Faculty Scheme awarded to Dr. Surajit Paul (code: IF-12/PH-44). S. Salunkhe wants to thank "Bhartratan JRD Tata Gunwant Sanshodhak Shishyavruti Yojna" for providing doctoral fellowship. PG acknowledges Council of Scientific and Industrial Research (CSIR) for providing senior research fellowship (CSIR$\mathrm{SRF}$ ) for supporting his $\mathrm{PhD}$ work. We are thankful to Dr. Neelam Panwar of ARIES, Naitital for her valuable suggestion regarding optical and UV image interpretation. SP and PG would like to thank The Inter-University Centre for Astronomy and Astrophysics (IUCAA) for providing the HPC facility. Computations described in this work were performed using the Enzo code (http://enzo.googlecode.com), which is the product of a collaborative effort of scientists at many universities and national laboratories and data analysis of the simulation is done with the yt-tools (http://yt-project.org).

\section{References}

Bagchi, J., Durret, F., Neto, G. B. L., \& Paul, S. 2006, Science, 314, 791 Blandford, R., \& Eichler, D. 1987, Phys. Rep., 154, 1

Böhringer, H., Voges, W., Huchra, J. P., et al. 2000, ApJS, 129, 435

Bonafede, A., Brüggen, M., van Weeren, R., et al. 2012, MNRAS, 426, 40

Bryan, G. L., Norman, M. L., O'Shea, B. W., et al. 2014, ApJS, 211, 19

Cen, R. 1992, ApJS, 78, 341

Clerc, N., Merloni, A., Zhang, Y.-Y., et al. 2016, MNRAS, 463, 4490

Colafrancesco, S., Marchegiani, P., \& Paulo, C. M. 2017, MNRAS, 471, 4747

de Gasperin, F., van Weeren, R. J., Brüggen, M., et al. 2014, MNRAS, 444, 3130 de Gasperin, F., Ogrean, G. A., van Weeren, R. J., et al. 2015, MNRAS, 448, 2197 de Gasperin, F., Intema, H. T., Ridl, J., et al. 2017, A\&A, 597, A15 de Gouveia dal Pino, E. M., \& Lazarian, A. 2005, A\&A, 441, 845 Drury, L. O. 1983, Rep. Progr. Phys., 46, 973

Dwarakanath, K. S., Parekh, V., Kale, R., \& George, L. T. 2018, MNRAS, 477, 957

Eisenstein, D. J., \& Hu, W. 1999, ApJ, 511, 5

Enßlin, T. A. 2006, Science, 314, 772

Enßlin, T. A., \& Gopal-Krishna 2001, A\&A, 366, 26

Enßlin, T. A., Biermann, P. L., Klein, U., \& Kohle, S. 1998, A\&A, 332, 395

Fang, K., \& Linden, T. 2016, JCAP, 10, 004

Ferland, G. J., Chatzikos, M., Guzmán, F., et al. 2017, Rev. Mex. Astron. Astrofis., 53, 385

Feretti, L., Giovannini, G., Govoni, F., \& Murgia, M. 2012, A\&ARv, 20, 54

Gal-Yam, A., Maoz, D., Guhathakurta, P., \& Filippenko, A. V. 2008, ApJ, 680, 550

Gilmour, R., Gray, M. E., Almaini, O., et al. 2007, MNRAS, 380, 1467

Govoni, F., \& Feretti, L. 2004, Int. J. Mod. Phys. D, 13, 1549

Hoeft, M., Brüggen, M., \& Yepes, G. 2004, MNRAS, 347, 389

Hong, S. E., Kang, H., \& Ryu, D. 2015, ApJ, 812, 49

Iapichino, L., \& Brüggen, M. 2012, MNRAS, 423, 2781

Jamrozy, M., Klein, U., Mack, K.-H., Gregorini, L., \& Parma, P. 2004, A\&A, 427, 79

Jubelgas, M., Springel, V., Enßlin, T., \& Pfrommer, C. 2008, A\&A, 481, 33

Kaiser, N. 1986, MNRAS, 222, 323

Kale, R., Wik, D. R., Giacintucci, S., et al. 2017, MNRAS, 472, 940

Kang, H., Ryu, D., \& Jones, T. W. 1996, ApJ, 456, 422

Kang, H., Ryu, D., \& Jones, T. W. 2012, ApJ, 756, 97

Kang, H., Ryu, D., \& Jones, T. W. 2017, ApJ, 840, 42

Kim, J.-Y., \& Trippe, S. 2014, J. Korean Astron. Soc., 47, 195

Komatsu, E., Dunkley, J., Nolta, M. R., et al. 2009, ApJS, 180, 330

Lara, L., Giovannini, G., Cotton, W. D., et al. 2004, A\&A, 421, 899

Lovisari, L., Reiprich, T. H., \& Schellenberger, G. 2015, A\&A, 573, A118

Mantz, A. B., Allen, S. W., Morris, R. G., \& von der Linden, A. 2018, MNRAS, 473, 3072

Paul, S. 2012, J. Phys. Conf. Ser., 405, 012026

Paul, S., Iapichino, L., Miniati, F., Bagchi, J., \& Mannheim, K. 2011, ApJ, 726, 17

Paul, S., John, R. S., Gupta, P., \& Kumar, H. 2017, MNRAS, 471, 2

Paul, S., Gupta, P., John, R. S., \& Pubjabi, V. 2018, ApJ, submitted [arXiv:1803.10764]

Paul, S., Salunkhe, S., Datta, A., \& Intema, H. T. 2019, MNRAS, 489, 446

Planck Collaboration XXIV. 2016, A\&A, 594, A27

Sarazin, C. L. 1988, Cambridge Astrophysics Series (Cambridge: Cambridge University Press)

Sarazin, C. L. 2002, Merging Processes in Galaxy Clusters, 272, 1

Sarazin, C. L., \& White, III., R. E. 1987, ApJ, 320, 32

Sharon, K., Gal-Yam, A., Maoz, D., Filippenko, A. V., \& Guhathakurta, P. 2007, ApJ, 660, 1165

Shimwell, T. W., Tasse, C., Hardcastle, M. J., et al. 2019, A\&A, 622, A1

Sivakoff, G. R., Martini, P., Zabludoff, A. I., Kelson, D. D., \& Mulchaey, J. S. 2008, ApJ, 682, 803

Strobel, N. V., Hodge, P., \& Kennicutt, R. C. 1991, ApJ, 383, 148

Stroe, A., Harwood, J. J., Hardcastle, M. J., \& Röttgering, H. J. A. 2014, MNRAS, 445, 1213

Subramanian, K. 1998, MNRAS, 294, 718

Subramanian, K., Shukurov, A., \& Haugen, N. E. L. 2006, MNRAS, 366, 1437

Turk, M. J., Smith, B. D., Oishi, J. S., et al. 2011, ApJS, 192, 9

van Weeren, R. J., de Gasperin, F., Akamatsu, H., et al. 2019, Space Sci. Rev., 215,16

Vazza, F., Brunetti, G., \& Gheller, C. 2009, MNRAS, 395, 1333 


\section{Appendix A: Modelling of cluster radio emission through cosmological simulations}

A cosmological simulation for a merging cluster was performed using ENZo, an $\mathrm{N}$-body plus hydrodynamic, Adaptive Mesh Refinement (AMR) code (Bryan et al. 2014). A flat $\Lambda$ CDM background cosmology with $\Omega_{\Lambda}=0.7257, \Omega_{\mathrm{m}}=0.2743$, $\Omega_{\mathrm{b}}=0.0458, h=0.702$ and primordial power spectrum normalisation $\sigma_{8}=0.816$ derived from WMAP (Komatsu et al. 2009) were used. The simulation was initialised at redshift $z=60$ using the Eisenstein \& Hu (1999) transfer function, and evolved up to redshift $z=0$. An ideal equation of state was used for the gas, with $\gamma=5 / 3$. Since the emergence and propagation of shocks are the most important events in this study, we captured the shocks very efficiently and resolved the grids adaptively wherever the shock was generated and passed by using the method described in Vazza et al. (2009). In order to capture the correct energy distribution of the ICM, radiative cooling (Sarazin \& White 1987) and a star-formation feedback scheme were applied (Cen 1992). Radio emission modelling was done as the post-processing numerical analysis by using the yt-tools (Turk et al. 2011). A detailed description of the simulation can be found in Paul et al. (2018).

Cosmological simulations were performed to create a sky realisation with a linear scale of $128 \mathrm{Mpc}(1+z)^{-1} h^{-1}$ [i.e. having a co-moving volume of $\left(128 \mathrm{Mpc} h^{-1}\right)^{3}$ ] with 0.3 million particles and $64^{3}$ grids at the root grid level. We also introduced two child grids, and inside the innermost child grid of $\left(32 \mathrm{Mpc} h^{-1}\right)^{3}$ co-moving volume, we implemented four levels of AMR, based on both over-densities and the shock strength. With this set-up, we achieved about $30 \mathrm{kpc} h^{-1}$ co-moving spatial resolution and mass resolution of about $10^{8} M_{\odot}$ at the highest resolved grids. For further details on the simulations and resolution studies of our numerical schemes, readers should refer to Paul et al. (2017, 2018).

\section{A.1. Radio emission modelling}

Radio emission takes place in a magnetised medium if relativistic charged particles (usually the electrons) are available. The ICM is known to host a magnetic field of $\mu \mathrm{G}$ order (Govoni \& Feretti 2004) that can be achieved through a turbulent dynamo model applied to a primordial seed magnetic field (Subramanian et al. 2006). But, the model is highly dependent on the degree of turbulence in the ICM. A saturation of magnetisation can be obtained once the turbulence fully develops in the usually Kolmogorov-type medium, meaning $E(k) \propto k^{-5 / 3}$ (where $k$ is wave number, and $E(k)$ is the energy per unit volume in $k$-space). Therefore, the equipartition is considered between magnetic energy density $\frac{B^{2}}{8 \pi}$ and the turbulent kinetic energy density $\rho \epsilon_{\text {turb }}$ in this condition (Subramanian 1998). A detailed description and the physical verification of the model can be found in Paul et al. (2018).

It is well known that the thermally-distributed ICM particles, after shock acceleration (through DSA), or turbulent reacceleration (TRA), produce a power-law energy distribution, $\left(\frac{\mathrm{d} n_{\mathrm{e}}}{\mathrm{d} E_{\mathrm{e}}}\right) \propto E^{-\delta}$

where $\delta$ is the spectral index of the injected electron energy. For DSA, the value is two or more steeper (Drury 1983). For TRA, the electron energy power law, with a simple assumption, would be to consider a fully-developed Kolmogorov-type turbulence, meaning $\delta$ to be $\frac{5}{2}$ (de Gouveia dal Pino \& Lazarian 2005; Fang \& Linden 2016).

Synchrotron radio emission is thus calculated using the standard synchrotron emission formula:

$\frac{\mathrm{d}^{2} P\left(v_{\mathrm{obs}}\right)}{\mathrm{d} V \mathrm{~d} v}=\frac{\sqrt{3} e^{3} B}{8 m_{\mathrm{e}} c^{2}} \int_{E_{\min }}^{E_{\max }} \mathrm{d} E_{\mathrm{e}} F\left(\frac{v_{\mathrm{obs}}}{v_{\mathrm{c}}}\right)\left(\frac{\mathrm{d} n_{\mathrm{e}}}{\mathrm{d} E_{\mathrm{e}}}\right)_{\mathrm{inj},}$

where $F(x)=x \int_{x}^{\infty} K_{5 / 3}\left(x^{\prime}\right) \mathrm{d} x^{\prime}$ is the synchrotron function, $K_{5 / 3}$ is the modified Bessel function, and $v_{\mathrm{c}}$ is the critical frequency of synchrotron emission,

$v_{c}=3 \gamma^{2} e B /\left(4 \pi m_{\mathrm{e}}\right)=1.6(B / 1 \mu \mathrm{G})\left(E_{\mathrm{e}} / 10 \mathrm{GeV}\right)^{2} \mathrm{GHz}$

$\frac{\mathrm{d} n_{\mathrm{e}}}{\mathrm{d} E_{\mathrm{e}}}$ is the injected electron energy spectrum determined either by DSA (Hong et al. 2015), or the TRA mechanism (Fang \& Linden 2016). Here, we did not include the synchrotron ageing model, which may lead to a slightly different spectrum of radio emission at low frequencies. For a detailed account of the computation of radio emissions for this study, see Paul et al. (2018).

\section{A.2. A cluster merger scenario}

A cluster merger scenario from our cosmological simulation is plotted in Fig. A.1. The columns from left to right represent specific phases of the galaxy cluster merger stage. The top row shows density maps. Figure A.1a clearly shows multiple groups approaching each other, meaning the pre-merger state. Figure A.1b shows that the groups merged into a single structure. Finally, Fig. A.1c shows that the structure becomes almost spheroidal during the post-merger relaxation phase. Further rows are for the same states as the above but representing temperature, vorticity magnitude $(\bar{\omega}=\nabla \times \bar{v}$, representing turbulence), and finally computed radio map, from top to bottom, respectively.

The temperature maps show, at pre-merger state, there is only a weak compression shock (most whitish part in the map). Merger shock launched at the centre of the panel e and after about $1.2 \mathrm{Gyr}$ of travelling through the cluster medium, crosses little more than an Mpc (see panel f) to reach the cluster periphery, similar to the observed cluster Abell 1697, though twosided. In the vorticity map in panel $\mathrm{i}$, one may notice that the turbulence is generated behind the shock front, as well as within a long trail of wake turbulence almost reaching the cluster centre (encircled by white dashed line), specifically for the shock on the right side of the image. The computed radio emission for the same (in panel 1) shows a long radio emission trail (encircled by black dashed line) behind the shock (simulated radio relic), generated due to turbulent re-acceleration from the wake turbulence. 

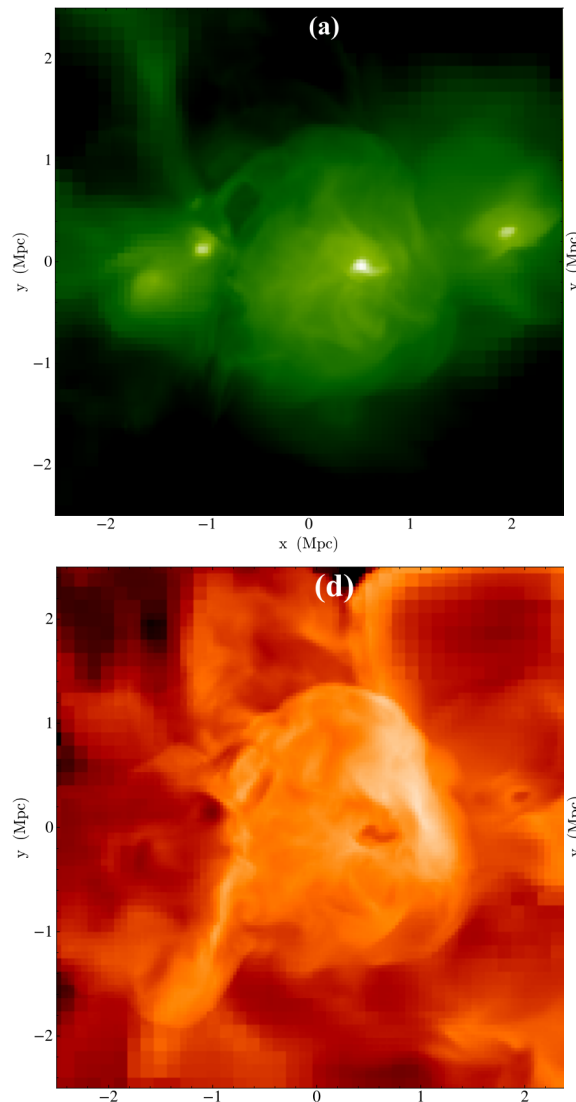

$\mathrm{x}(\mathrm{Mpc})$
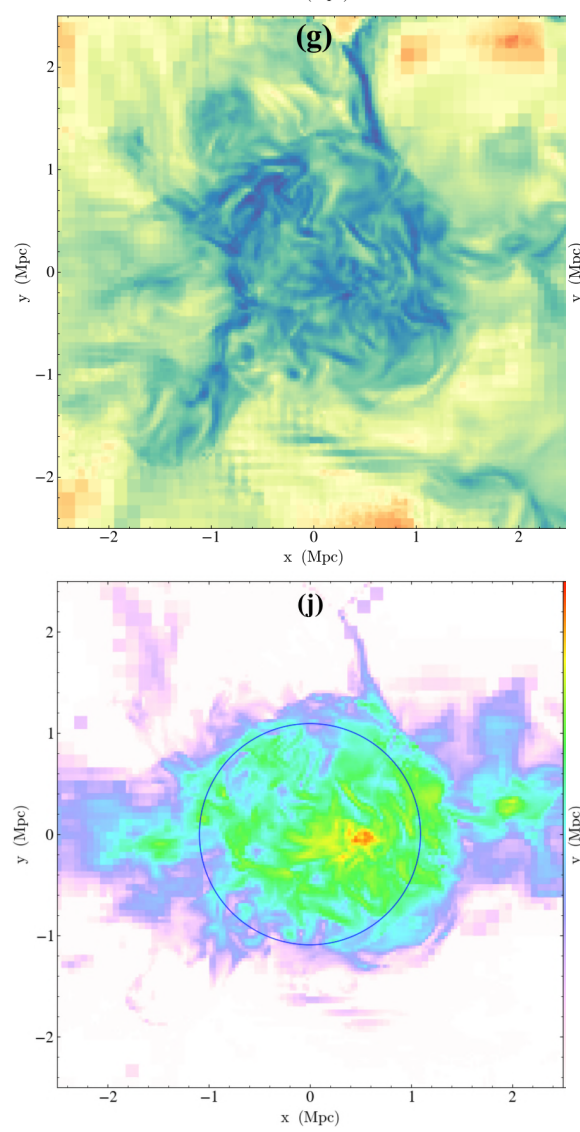
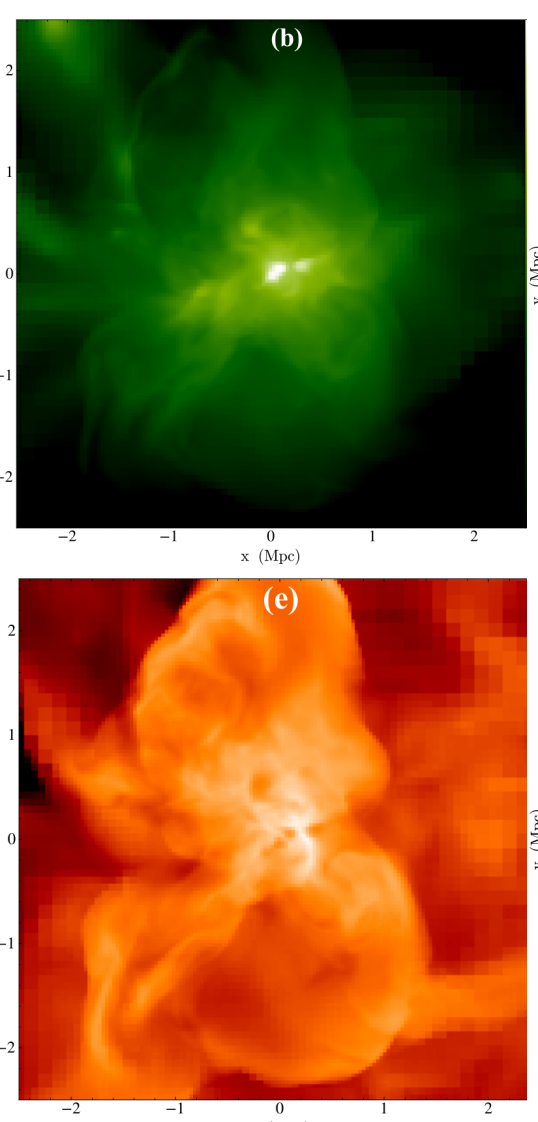

$\mathrm{x}$ (Mpc)
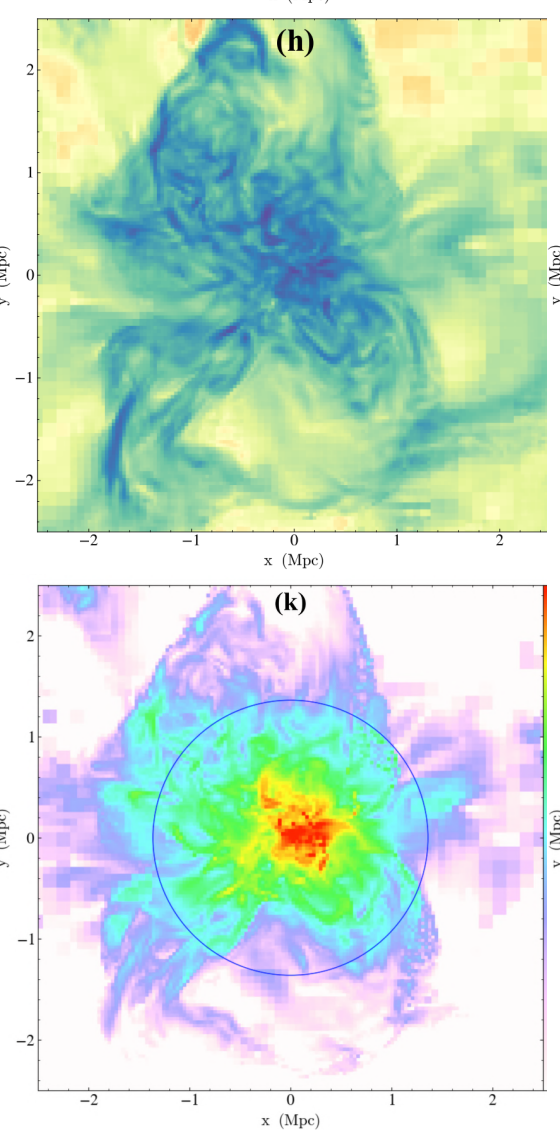
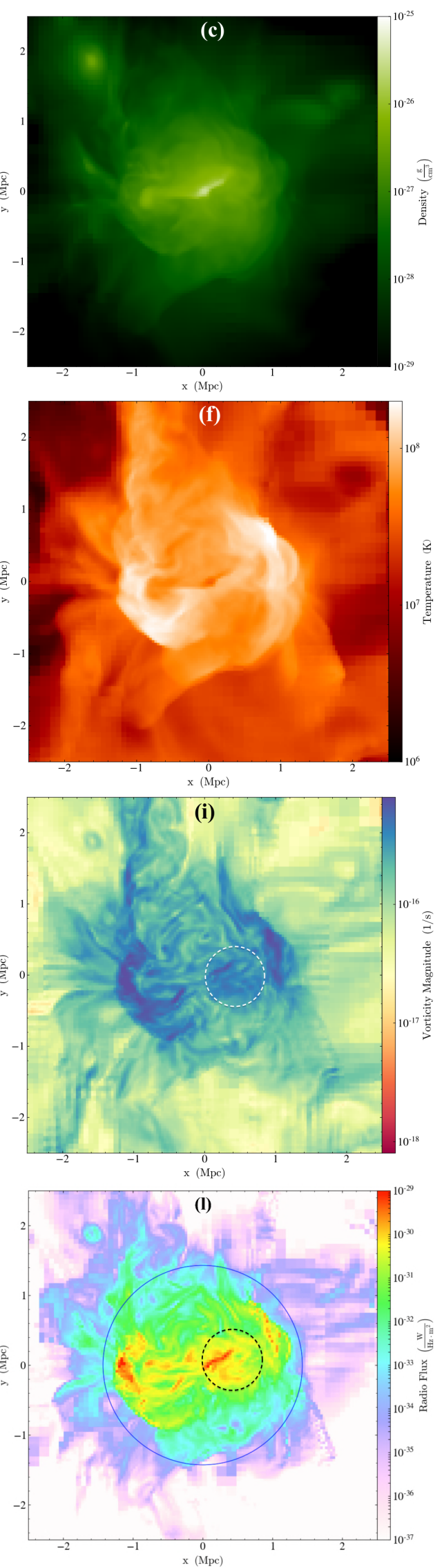

Fig. A.1. Each panel is a slice plot of a $5 \times 5 \mathrm{Mpc}^{2}$ area focused on a time series of a cluster merger event. Density (first row), temperature (second row), vorticity magnitude (third row) and radio emission maps of simulated cluster at a pre-merger (first column), merger (second column) and postmerger (third column) state. The white and black dashed circle in panels $i$ and $l$ respectively are wake turbulence and corresponding radio emission from TRA electrons. 